\title{
NONEXPONENTIAL ASYMPTOTICS FOR THE SOLUTIONS OF RENEWAL EQUATIONS, WITH APPLICATIONS
}

\author{
CHUANCUN YIN $^{* * *}$ AND \\ JUNSHENG ZHAO, ${ }^{*}$ Qufu Normal University
}

\begin{abstract}
Nonexponential asymptotics for solutions of two specific defective renewal equations are obtained. These include the special cases of asymptotics for a compound geometric distribution and the convolution of a compound geometric distribution with a distribution function. As applications of these results, we study the asymptotic behavior of the demographic birth rate of females, the perpetual put option in mathematics of finance, and the renewal function for terminating renewal processes.

Keywords: Asymptotics; defective renewal equation; deterministic population model; compound geometric distribution; heavy-tailed distribution; perpetual put option; renewal function

2000 Mathematics Subject Classification: Primary 60K05
\end{abstract}

Secondary $60 \mathrm{~K} 10 ; 60 \mathrm{~K} 30$

\section{Introduction}

Throughout this paper, all distributions are supported on $[0, \infty)$. We denote the tail of distribution $F$ by $\bar{F}=1-F$ and the moment generating function of $F$ by $m_{F}(s)=\int_{0}^{\infty} \mathrm{e}^{s x} \mathrm{~d} F(x)$. For two functions $f_{1}$ and $f_{2}, f_{1}(x) \sim f_{2}(x)$ means that $\lim _{x \rightarrow \infty}\left(f_{1}(x) / f_{2}(x)\right)=1$ and $f_{1}(x)=o\left(f_{2}(x)\right)$ means that $\lim _{x \rightarrow \infty}\left(f_{1}(x) / f_{2}(x)\right)=0$.

We consider the renewal equation

$$
Z(x)=g(x)+\alpha \int_{0}^{x} Z(x-y) \mathrm{d} F(y), \quad x \geq 0,
$$

where $\alpha>0, F(y)=1-\bar{F}(y)$ is a proper probability distribution function with $F(0)=0$, and $g(x) \geq 0$ is locally bounded. The renewal equation is called proper, defective, or excessive, according to whether $\alpha$ is equal to one, is less than one, or is bigger than one, respectively. In applications, the typical situation is that an unknown function $Z$ satisfies a renewal equation of the form (1.1), where $g, \alpha$, and $F$ are known. Based on this, we want to draw conclusions about the function $Z$.

The only solution $Z(x)$ to (1.1) which is vanishing for $x<0$ and bounded on finite intervals is given by

$$
Z(x)=g(x)+\sum_{k=1}^{\infty} \alpha^{k} \int_{0}^{x} g(x-y) \mathrm{d} F^{* k}(y), \quad x \geq 0,
$$

Received 19 September 2005; revision received 6 March 2006.

* Postal address: Department of Mathematics, Qufu Normal University, Qufu, 273165, Shandong, P. R. China.

** Email address: ccyin@mail.qfnu.edu.cn 
(see, e.g. Rolski et al. (1999, Lemma 6.1.2)), where $F^{* k}$ is the distribution function of the $k$-fold convolution of $F$ with itself.

The renewal equation of the form (1.1) arises in many applied probability models, such as branching processes, queueing theory, insurance ruin theory, reliability theory, demography, and so on. However, it is rarely soluble in closed form. Hence, there has been great interest in estimating the solution. The main purpose of this paper is to study the asymptotic behavior of the solution $Z(x)$ of (1.2) for large $x$. Set $K(\lambda)=\alpha \int_{0}^{\infty} \mathrm{e}^{\lambda x} \mathrm{~d} F(x)$ and $l=\sup \{\lambda \geq 0: K(\lambda) \leq 1\}$. Since $F(0)=0$, we always have $l<\infty$. Only the following three cases can occur:

(i) $l>0$ and $K(l)=1$, the 'Cramér case',

(ii) $l=0$, the 'heavy-tailed case',

(iii) $l>0$ and $K(l)<1$, the 'intermediate case'.

It turns out that the asymptotic behavior of $Z(x)$ heavily depends on the case which applies. For case (i), if $F$ is a nonlattice distribution and $\mathrm{e}^{l x} g(x)$ is directly Riemann integrable, then the famous Cramér-Lundberg estimate states that

$$
Z(x) \sim \frac{\int_{0}^{\infty} \mathrm{e}^{l y} g(y) \mathrm{d} y}{\alpha \int_{0}^{\infty} y \mathrm{e}^{l y} \mathrm{~d} F(y)} \mathrm{e}^{-l x},
$$

(see, e.g. Feller (1971)). Obviously, if $\alpha \geq 1$ then we can always find $l$ satisfying condition (i), but if $\alpha<1$ then there may be no such number and, thus, there is no exponential CramérLundberg estimate for $Z(x)$. Asmussen (1998) investigated the asymptotic behavior of $Z(x)$ under the conditions that $g$ is integrable, $g(x) \sim b(x)$ for some subexponential density $b$, and $F$ has a subexponential density. Sharp upper and lower bounds on the solution $Z(x)$ to (1.1) have been derived in various situations; see Willmot et al. (2001) and references therein for details. In particular, if $g(x)=F(x)$ then (1.2) is a compound geometric distribution which has been studied by many authors (see, e.g. Embrechts et al. (1979), Embrechts and Goldie (1982), and Cline (1987)). If $g(x)=G(x)$, a distribution function supported on $[0, \infty)$, then (1.2) is the convolution of a compound geometric distribution with $G$. For this case, the asymptotic behavior and bounds of $\bar{Z}(x)$ have been studied by Cai and Garrido (2002) and Cai and Tang (2004).

In this paper, we investigate the nonexponential estimates of $Z(x)$ for some functions $g(x)$ in the cases (ii) and (iii), and then discuss applications of these results. In the sequel, we always assume that $\alpha<1$.

\section{Preliminaries}

A random variable $X$ (or its distribution function $F$ ) is said to be heavy-tailed on the right if $\mathrm{E}^{r X}=\infty$ for any $r>0$. Heavy-tailed distributions have been the focus of study of many researchers in finance and insurance in recent years; see Embrechts et al. (1997) for a review.

A distribution function $F$ is said to belong to the class $\mathcal{L}$ of long-tailed distributions if $\lim _{x \rightarrow \infty}(\bar{F}(x-y) / \bar{F}(x))=1$ for every $y>0$ (or, equivalently, for some $y>0$ ); $F$ is said to belong to the subexponential class $\&$ if $\lim _{x \rightarrow \infty}\left(\overline{F^{* n}}(x) / \bar{F}(x)\right)=n$ for any $n \geq 2$ (or, equivalently, for $n=2$ ); and $F$ is said to belong to the class $\mathscr{D}$ of distribution functions with dominatedly varying tails if $\lim _{\sup _{x \rightarrow \infty}}(\bar{F}(x y) / \bar{F}(x))<\infty$ for every $y \in(0,1)$ (or, equivalently, for some $y \in(0,1)$ ). It is well known that $\mathscr{D} \cap \mathcal{L} \subset s \subset \mathcal{L}$ (see, e.g. Embrechts et al. (1997)). 
We say that $F \in \delta(\gamma), \gamma \geq 0$, if and only if

$$
\begin{gathered}
\lim _{x \rightarrow \infty} \frac{\overline{F^{* 2}}(x)}{\bar{F}(x)}=2 m_{F}(\gamma)<\infty, \\
\lim _{x \rightarrow \infty} \frac{\bar{F}(x-y)}{\bar{F}(x)}=\mathrm{e}^{\gamma y},
\end{gathered}
$$

for all real $y$. We say that $F \in \mathcal{L}(\gamma)$ if and only if $F$ satisfies (2.1). Note that $\delta(0)=\delta$, the class of subexponential distribution functions, and $\mathcal{L}(0)=\mathscr{L}$, the class of long-tailed distribution functions. Distributions in $\delta(\gamma), \gamma>0$, are dominated by an exponential, i.e. $\lim _{x \rightarrow \infty} \mathrm{e}^{\gamma x} \bar{F}(x)=0$. One way to construct densities whose distributions are in $\delta(\gamma)$ for $\gamma>0$ is to multiply densities whose distributions are in $\delta$ by negative exponentials. A typical example in $\delta(\gamma)$ is $F$ with $F^{\prime}(x) \sim x^{-b} \mathrm{e}^{-\gamma x}$ as $x \rightarrow \infty$, with $b>1$. We refer the reader to Chover et al. (1973a), Embrechts and Veraverbeke (1982), Embrechts and Goldie (1982), and Klüppelberg (1989) for nice reviews of the classes $\delta(\gamma)$ and $\mathcal{L}(\gamma)$, among others.

We now summarize some of the results about the classes of distributions above which will be used in the sequel.

Lemma 2.1. For $\gamma \geq 0$, if $F_{1} \in \mathcal{L}(\gamma), F_{2} \in s(\gamma)$, and $\sup _{x \geq 0}\left(\overline{F_{1}}(x) / \overline{F_{2}}(x)\right)<\infty$, then

$$
\overline{F_{1} * F_{2}}(x) \sim m_{F_{1}}(\gamma) \overline{F_{2}}(x)+m_{F_{2}}(\gamma) \overline{F_{1}}(x) .
$$

Proof. For the case $\gamma=0$, see Embrechts and Goldie (1980); for the case $\gamma>0$, see Cline (1986).

Remark 2.1. For the case $\gamma=0$, if the boundedness of $\overline{F_{1}}(x) / \overline{F_{2}}(x)$ in Lemma 2.1, is strengthened to the requirement $\overline{F_{1}}(x)=o\left(\overline{F_{2}}(x)\right)$, then the condition $F_{1} \in \mathcal{L}$ can be omitted (see Embrechts et al. (1979, Proposition 1)).

Lemma 2.2. For $\gamma \geq 0$, if $F \in s(\gamma)$ then,

(i) for all integers $n \geq 1, \lim _{x \rightarrow \infty}\left(\overline{F^{* n}}(x) / \bar{F}(x)\right)=n\left(m_{F}(\gamma)\right)^{n-1}$,

(ii) for any $\varepsilon>0$, there exists a finite constant $K_{\varepsilon}$ such that

$$
\overline{F^{* n}}(x) \leq K_{\varepsilon}\left(m_{F}(\gamma)+\varepsilon\right)^{n-1} \bar{F}(x)
$$

for all $n \geq 2$ and all $x \geq 0$.

Proof. For the proof of the case $\gamma=0$, see Embrechts et al. (1997); for the case $\gamma>0$, see Chover et al. (1973a), (1973b).

Lemma 2.3. (i) (Cline (1987, Theorem 2.13).) Assume that $F \in \mathcal{L}(\gamma), \quad \gamma>0$. Let $\left\{\lambda_{n}\right\}$ be a sequence of nonnegative coefficients such that $\lambda_{j}>0$ for some $j>1$ and $\sum_{n=0}^{\infty} \lambda_{n}\left(m_{F}(\gamma)+\varepsilon\right)^{n}<\infty$ for some $\varepsilon>0$. Let $H=\sum_{n=0}^{\infty} \lambda_{n} F^{* n}$. The following statements are equivalent:

(a) $F \in \delta(\gamma)$,

(b) $\bar{H} \sim c \bar{F}$ for $c=\sum_{n=1}^{\infty} n \lambda_{n}\left(m_{F}(\gamma)\right)^{n-1}$. 
(ii) (Embrechts et al. (1979, Lemma 3).) Suppose that $\alpha \in(0,1)$ and $G$ is a proper distribution function on $[0, \infty)$. If $F(x)=(1-\alpha) \sum_{n=0}^{\infty} \alpha^{n} G^{* n}(x)$ then the following assertions are equivalent:

(c) $F \in 8$,

(d) $G \in \&$,

(e) $\bar{F} \sim(\alpha /(1-\alpha)) \bar{G}$.

Lemma 2.4. (Cai and Tang (2004).) If $F_{1}, F_{2} \in \mathcal{D} \cap \mathcal{L}$ then

$$
F_{1} * F_{2} \in \mathscr{D} \cap \mathcal{L} \text { and } \overline{F_{1} * F_{2}}(x) \sim \overline{F_{1}}(x)+\overline{F_{2}}(x) .
$$

\section{Asymptotic behavior of the solutions}

Theorem 3.1. Consider the renewal equation (1.1). Assume that $g(x) \geq 0$ is right-continuous and decreasing to 0 . Let $G(x)=(g(0)-g(x)) / g(0), x \geq 0$.

(i) If $F \in \&, G \in \&$, and $\sup _{x}(\bar{F}(x) / \bar{G}(x))<\infty$, then

$$
Z(x) \sim \frac{g(x)}{1-\alpha} .
$$

(ii) If $F \in \varsigma$ and $\bar{G}(x)=o(\bar{F}(x))$, then

$$
Z(x)=o(\bar{F}(x))
$$

(iii) If $\gamma>0, \alpha m_{F}(\gamma)<1, F \in \delta(\gamma), G \in \delta(\gamma)$, and $\sup _{x}(\bar{F}(x) / \bar{G}(x))<\infty$, then

$$
Z(x) \sim \frac{\alpha g(0)\left(m_{G}(\gamma)-1\right)}{\left(1-\alpha m_{F}(\gamma)\right)^{2}} \bar{F}(x)+\frac{g(x)}{1-\alpha m_{F}(\gamma)} .
$$

(iv) If $\gamma>0, \alpha m_{F}(\gamma)<1, F \in \delta(\gamma), G \in \delta(\gamma)$, and $\bar{G}(x)=o(\bar{F}(x))$, then

$$
Z(x) \sim \frac{\alpha g(0)\left(m_{G}(\gamma)-1\right)}{\left(1-\alpha m_{F}(\gamma)\right)^{2}} \bar{F}(x) .
$$

Proof. (i) Observe that $G$ is a proper distribution function on $[0, \infty)$ satisfying $G(0)=0$ with right-tail $\bar{G}(x)=g(x) / g(0), x \geq 0$. It follows from (1.2) that $Z(x)$ can be expressed as

$$
\begin{aligned}
Z(x) & =\frac{g(0)}{1-\alpha} \int_{0}^{x} \bar{G}(x-y) \mathrm{d} U_{0}(y) \\
& =\frac{g(0)}{1-\alpha}\left(\overline{G * U_{0}}(x)-\overline{U_{0}}(x)\right),
\end{aligned}
$$

where

$$
U_{0}(x)=(1-\alpha) \sum_{n=0}^{\infty} \alpha^{n} F^{* n}(x) .
$$

By Lemma 2.3(ii) or Cai and Tang (2004, Proposition 1.1(c)),

$$
\overline{U_{0}}(x) \sim \frac{\alpha}{1-\alpha} \bar{F}(x),
$$


which implies that $U_{0} \in \delta$, since $\&$ is closed with respect to tail equivalence (see, e.g. Teugels (1975)). Using Lemma 2.2(ii) and the condition $\sup _{x}(\bar{F}(x) / \bar{G}(x))<\infty$, we find that $\sup _{x}\left(\overline{U_{0}}(x) / \bar{G}(x)\right)<\infty$. Thus, it follows from Lemma 2.1 that $\overline{G * U_{0}}(x) \sim \bar{G}(x)+\overline{U_{0}}(x)$, which, together with (3.5), gives

$$
\begin{aligned}
\frac{Z(x)}{\bar{G}(x)} & =\frac{g(0)}{1-\alpha}\left(\frac{\bar{G}(x)+\overline{U_{0}}(x)+o\left(\bar{G}(x)+\overline{U_{0}}(x)\right)}{\bar{G}(x)}-\frac{\overline{U_{0}}(x)}{\bar{G}(x)}\right) \\
& =\frac{g(0)}{1-\alpha}\left(1+\frac{o\left(\bar{G}(x)+\overline{U_{0}}(x)\right)}{\bar{G}(x)+\overline{U_{0}}(x)}\left(1+\frac{\overline{U_{0}}(x)}{\bar{G}(x)}\right)\right) \\
& \rightarrow \frac{g(0)}{1-\alpha} .
\end{aligned}
$$

Since $g(x)=g(0) \bar{G}(x),(3.1)$ follows.

(ii) The condition $\bar{G}(x)=o(\bar{F}(x))$ implies that $\bar{G}(x)=o\left(\overline{U_{0}}(x)\right)$ since $\overline{U_{0}}(x) \sim$ $(\alpha /(1-\alpha)) \bar{F}(x)$. If $F \in \delta$ then, by Lemma 2.1 and Remark 2.1 or using Cai and Garrido (2002, Theorem 3.3), we have $\overline{G * U_{0}}(x) \sim \overline{U_{0}}(x)$, which gives (3.2) by using (3.5).

Similarly, (3.3) and (3.4) follow from Lemma 2.1 and the fact that (by Lemma 2.3(i))

$$
\overline{U_{0}}(x) \sim \frac{\alpha(1-\alpha)}{\left(1-\alpha m_{F}(\gamma)\right)^{2}} \bar{F}(x),
$$

which implies that $U_{0} \in \delta(\gamma)$ since $\delta(\gamma)$ is closed with respect to tail equivalence (see Embrechts and Goldie (1982, Theorem 2.7)). This ends the proof of Theorem 3.1.

Theorem 3.2. Consider the renewal equation (1.1). Suppose that $g(x) \geq 0$ with $g(0)=0$ is a right-continuous and nondecreasing function such that $\lim _{x \rightarrow \infty} g(x)=g(\infty)<\infty$. Let $P(x)=g(x) / g(\infty), x \geq 0$.

(i) If $F \in \delta, P \in \delta$, and $\sup _{x}(\bar{F}(x) / \bar{P}(x))<\infty$, then

$$
Z(\infty)-Z(x) \sim \frac{g(\infty)-g(x)}{1-\alpha}+\frac{\alpha g(\infty)}{(1-\alpha)^{2}} \bar{F}(x) .
$$

In particular, if $g(\infty)=1$ then $\bar{Z}(x) \sim \bar{g}(x) /(1-\alpha)+\left(\alpha /(1-\alpha)^{2}\right) \bar{F}(x)$.

(ii) If $F \in \&$ and $\bar{P}(x)=o(\bar{F}(x))$, then

$$
Z(\infty)-Z(x) \sim \frac{\alpha g(\infty)}{(1-\alpha)^{2}} \bar{F}(x) .
$$

In particular, if $g(\infty)=1$ then $\bar{Z}(x) \sim\left(\alpha /(1-\alpha)^{2}\right) \bar{F}(x)$.

(iii) If $\gamma>0, \alpha m_{F}(\gamma)<1, F \in \delta(\gamma), P \in \delta(\gamma)$, and $\sup _{x}(\bar{F}(x) / \bar{P}(x))<\infty$, then

$$
Z(\infty)-Z(x) \sim \frac{g(\infty)-g(x)}{1-\alpha m_{F}(\gamma)}+\frac{\alpha g(\infty) m_{P}(\gamma)}{\left(1-\alpha m_{F}(\gamma)\right)^{2}} \bar{F}(x) .
$$

In particular, if $g(\infty)=1$ then

$$
\bar{Z}(x) \sim \frac{1}{1-\alpha m_{F}(\gamma)} \bar{g}(x)+\frac{\alpha m_{g}(\gamma)}{\left(1-\alpha m_{F}(\gamma)\right)^{2}} \bar{F}(x) .
$$


(iv) If $\gamma>0, \alpha m_{F}(\gamma)<1, F \in \delta(\gamma), P \in \delta(\gamma)$, and $\bar{P}(x)=o(\bar{F}(x))$, then

$$
Z(\infty)-Z(x) \sim \frac{\alpha g(\infty) m_{P}(\gamma)}{\left(1-\alpha m_{F}(\gamma)\right)^{2}} \bar{F}(x) .
$$

In particular, if $g(\infty)=1$ then $\bar{Z}(x) \sim\left(\alpha m_{g}(\gamma) /\left(1-\alpha m_{F}(\gamma)\right)^{2}\right) \bar{F}(x)$.

Proof. (i) Clearly, $P$ is a proper distribution function on $[0, \infty)$ satisfying $P(0)=0$ with right-tail

$$
\bar{P}(x)=\frac{g(\infty)-g(x)}{g(\infty)} .
$$

It follows from (1.2) that the solution of (1.1) can be written as

$$
Z(x)=(1-\alpha)^{-1} g(\infty)\left(P * U_{0}\right)(x), \quad x \geq 0,
$$

where, as before, $U_{0}(x)=(1-\alpha) \sum_{n=0}^{\infty} \alpha^{n} F^{* n}(x)$. Thus,

$$
\frac{Z(x)}{Z(\infty)}=\left(P * U_{0}\right)(x), \quad x \geq 0,
$$

since $Z(\infty)=g(\infty)(1-\alpha)^{-1}$ by (3.7). As in the proof of Theorem 3.1(i), $U_{0} \in \&, \overline{U_{0}}(x) \sim$ $(\alpha /(1-\alpha)) \bar{F}(x)$, and $\sup _{x}\left(\overline{U_{0}}(x) / \bar{P}(x)\right)<\infty$. By Lemma 2.1 we obtain

$$
\overline{P * U_{0}}(x) \sim \bar{P}(x)+\overline{U_{0}}(x) \sim \bar{P}(x)+\frac{\alpha}{1-\alpha} \bar{F}(x),
$$

which, together with (3.8), gives (3.6).

The proof of part (ii) is virtually the same as that of Theorem 3.1(ii). We can prove parts (iii) and (iv) in a similar way to parts (i) and (ii). This ends the proof of Theorem 3.2.

Remark 3.1. We remark that the condition $\sup _{x}(\bar{F}(x) / \bar{P}(x))<\infty$ in Theorem 3.2 is weaker than the conditions used by Cai and Garrido (2002, Theorems 3.4, 3.5, and 4.1), and the asymptotic results of the above paper can be deduced from Theorem 3.2.

The following theorem does not assume a domination relationship between $F$ and $P$.

Theorem 3.3. Suppose that $g(x)$ satisfies the same conditions as in Theorem 3.2. If $F, P \in$ $\mathscr{D} \cap \mathcal{L}$, where $P(x)=g(x) / g(\infty)$, then

$$
Z(\infty)-Z(x) \sim \frac{g(\infty)-g(x)}{1-\alpha}+\frac{\alpha g(\infty)}{(1-\alpha)^{2}} \bar{F}(x) .
$$

In particular, if $g(\infty)=1$ then $\bar{Z}(x) \sim \bar{g}(x) /(1-\alpha)+\left(\alpha /(1-\alpha)^{2}\right) \bar{F}(x)$.

Proof. The proof is similar to that of Theorem 3.2, but using Lemma 2.4 instead of Lemma 2.1.

\section{Applications}

In this section, we study the asymptotic behavior of various renewal equations arising in applied probability models. Several examples from insurance ruin theory, branching processes, and queueing theory can be found in Willmot et al. (2001). We first consider an example from demography. 
Example 4.1. (Deterministic population model.) Let $B(t)$ denote the rate at which females are born at time $t$, i.e. $B(t) \mathrm{d} t$ is the number of female babies born between times $t$ and $t+\mathrm{d} t$. Given $B(t)$ for $t \leq 0$, project $B(t)$ for $t>0$. For this purpose, assume a survival function $s(x)$ and an age-specific force of birth $\beta(x), x>0$. Thus, $s(x)$ is the probability that a newly born female survives to age $x$, and $\beta(x) \mathrm{d} t$ is the number of female babies borne by a parent of age $x$ in a time interval of length $\mathrm{d} t$. Then $B(t)$ satisfies the following renewal equation (see Gerber (1979)):

$$
B(t)=\int_{t}^{\infty} B(t-x) s(x) \beta(x) \mathrm{d} x+\int_{0}^{t} B(t-x) s(x) \beta(x) \mathrm{d} x .
$$

Clearly, (4.1) is of the form (1.1) with $g(x)=\int_{x}^{\infty} B(x-y) s(y) \beta(y) \mathrm{d} y, \alpha=\int_{0}^{\infty} s(x) \beta(x) \mathrm{d} x$, and

$$
F(x)=\frac{\int_{0}^{x} s(y) \beta(y) \mathrm{d} y}{\int_{0}^{\infty} s(y) \beta(y) \mathrm{d} y} .
$$

If there exist positive constants $c_{i}$ such that $c_{1} \leq \beta(x) \leq c_{2}$ and $c_{3} \leq B(-x) \leq c_{4}$, for $x \geq 0$, then

$$
\frac{c_{1}}{c_{2}} \leq \frac{\bar{F}(x)}{\bar{S}(x)} \leq \frac{c_{2}}{c_{1}}
$$

for all $x \in[0, \infty)$ and $\sup _{x \geq 0}(\bar{F}(x) / \bar{G}(x))<\infty$, where

$$
\bar{S}(x)=\frac{\int_{x}^{\infty} s(y) \mathrm{d} y}{\int_{0}^{\infty} s(y) \mathrm{d} y}, \quad \bar{G}(x)=\frac{\int_{x}^{\infty} B(x-y) s(y) \beta(y) \mathrm{d} y}{\int_{0}^{\infty} B(x-y) s(y) \beta(y) \mathrm{d} y} .
$$

Thus, if $S \in \delta(\gamma)$ then $F \in \delta(\gamma)$ (see, e.g. Klüppelberg (1988, Theorem 2.1)). If, in addition, $\alpha m_{F}(\gamma)<1$, then the conditions of Theorem 3.1 are fulfilled. Consequently, Theorem 3.1 yields

$$
B(x) \sim \begin{cases}\frac{\int_{x}^{\infty} B(x-y) s(y) \beta(y) \mathrm{d} y}{1-\int_{0}^{\infty} s(y) \beta(y) \mathrm{d} y} & \text { if } \gamma=0, \\ \frac{\left(m_{G}(\gamma)-1\right) \int_{x}^{\infty} s(y) \beta(y) \mathrm{d} y \int_{0}^{\infty} B(-y) s(y) \beta(y) \mathrm{d} y}{\left(1-\alpha m_{F}(\gamma)\right)^{2}} & \\ +\frac{\int_{x}^{\infty} B(x-y) s(y) \beta(y) \mathrm{d} y}{1-\alpha m_{F}(\gamma)} & \text { if } \gamma>0 .\end{cases}
$$

The second example is from mathematics of finance.

Example 4.2. (Pricing a perpetual put option.) Let $S(t)$ denote the price at time $t \geq 0$ of a non-dividend-paying stock. We assume that the logarithm of the stock price process follows a process of the form $U(t)=u+c t-Z(t)$, where $c>0$ is constant and $\{Z(t)\}$ is a compound Poisson process specified by the Poisson parameter $\lambda>0$ and the distribution of the jump amounts. To simplify notation, we suppose that the latter is continuous with probability density $p(x), x \geq 0$. We further assume that the market is frictionless and risk-neutral, and that there is a constant risk-free force of interest, $r>0$.

We consider a perpetual put option with payoff function $\Pi(s)=\max (K-s, 0)$, where $K$ is the exercise price. We restrict ourselves to options where it is clear a priori that the optimal exercise strategy is a stopping time of the form $T_{L}=\inf \{t: S(t)<L\}$. Let

$$
V(s ; L)=\mathrm{E}\left[\mathrm{e}^{-r T_{L}} \Pi\left(S\left(T_{L}\right)\right) \mid S(0)=s\right], \quad s \geq L,
$$


denote the expected discounted value of the payoff if the initial stock price is $s$ and the strategy $T_{L}$ is applied. Then $V(s ; L)$ satisfies the equation (see Gerber and Shiu (1998))

$$
V(s ; L)=\int_{0}^{\ln (s / L)} V\left(s \mathrm{e}^{-y} ; L\right) h(y) \mathrm{d} y+\int_{\ln (s / L)}^{\infty} \Pi\left(s \mathrm{e}^{-y}\right) h(y) \mathrm{d} y, \quad s \geq L,
$$

where

$$
h(y)=\frac{\lambda}{c} \mathrm{e}^{y} \int_{y}^{\infty} \mathrm{e}^{-x} p(x) \mathrm{d} x .
$$

Replace $s$ by $x=\ln (s / L)$ and consider the function $W(x ; L)$, defined by $W(x ; L)=V(s ; L)$. Then $W$ satisfies the following defective renewal equation:

$$
W(x ; L)=\int_{0}^{x} W(x-y ; L) h(y) \mathrm{d} y+\int_{x}^{\infty} \Pi\left(L \mathrm{e}^{x-y}\right) h(y) \mathrm{d} y, \quad x \geq 0 .
$$

Clearly, (4.2) is of the form (1.1) with $g(x)=\int_{x}^{\infty} \Pi\left(L \mathrm{e}^{x-y}\right) h(y) \mathrm{d} y, \alpha=\int_{0}^{\infty} h(x) \mathrm{d} x$, and $F(x)=\left(\int_{0}^{\infty} h(x) \mathrm{d} x\right)^{-1} \int_{0}^{x} h(x) \mathrm{d} x$. Let $\bar{G}(x)=g(x) / g(0)$. If $F \in \delta(\gamma), \gamma \geq 0$, then it is not difficult to verify that

$$
\lim _{x \rightarrow \infty} \frac{\bar{F}(x)}{\bar{G}(x)}= \begin{cases}\frac{\int_{0}^{\infty} \Pi\left(L \mathrm{e}^{-y}\right) h(y) \mathrm{d} y}{\int_{0}^{\infty} h(y) \mathrm{d} y\left(K-\left(\gamma L \int_{0}^{\infty} h(y) \mathrm{d} y\right) /\left(1+\gamma \int_{0}^{\infty} h(y) \mathrm{d} y\right)\right)} & \text { if } \gamma>0 \\ \frac{\int_{0}^{\infty} \Pi\left(L \mathrm{e}^{-y}\right) h(y) \mathrm{d} y}{K \int_{0}^{\infty} h(y) \mathrm{d} y} & \text { if } \gamma=0 .\end{cases}
$$

Thus, $G \in \delta(\gamma)$ and $\sup _{u \geq 0}(\bar{F}(u) / \bar{G}(u))<\infty$, since $\delta(\gamma)$ is closed with respect to tail equivalence. Applying Theorem 3.1 yields the following results.

(i) For $\gamma=0$,

$$
W(x ; L) \sim \frac{\int_{x}^{\infty} \Pi\left(L \mathrm{e}^{x-y}\right) h(y) \mathrm{d} y}{1-\int_{0}^{\infty} h(y) \mathrm{d} y}, \quad \text { as } x \rightarrow \infty,
$$

or

$$
V(s ; L) \sim \frac{\int_{\ln (s / L)}^{\infty} \Pi\left(s \mathrm{e}^{-y}\right) h(y) \mathrm{d} y}{1-\int_{0}^{\infty} h(y) \mathrm{d} y}, \quad \text { as } s \rightarrow \infty .
$$

(ii) For $\gamma>0$,

$$
\begin{aligned}
W(x ; L) \sim & \frac{\left(m_{G}(\gamma)-1\right) \int_{0}^{\infty} \Pi\left(L \mathrm{e}^{-y}\right) h(y) \mathrm{d} y \int_{x}^{\infty} h(y) \mathrm{d} y}{\left(1-m_{F}(\gamma) \int_{0}^{\infty} h(y) \mathrm{d} y\right)^{2}} \\
& +\frac{\int_{x}^{\infty} \Pi\left(L \mathrm{e}^{x-y}\right) h(y) \mathrm{d} y}{1-m_{F}(\gamma) \int_{0}^{\infty} h(y) \mathrm{d} y}, \quad \text { as } x \rightarrow \infty,
\end{aligned}
$$

or

$$
\begin{aligned}
V(s ; L) \sim & \frac{\left(m_{G}(\gamma)-1\right) \int_{0}^{\infty} \Pi\left(L \mathrm{e}^{-y}\right) h(y) \mathrm{d} y \int_{\ln (s / L)}^{\infty} h(y) \mathrm{d} y}{\left(1-m_{F}(\gamma) \int_{0}^{\infty} h(y) \mathrm{d} y\right)^{2}} \\
& +\frac{\int_{\ln (s / L)}^{\infty} \Pi\left(s \mathrm{e}^{-y}\right) h(y) \mathrm{d} y}{1-m_{F}(\gamma) \int_{0}^{\infty} h(y) \mathrm{d} y}, \quad \text { as } s \rightarrow \infty .
\end{aligned}
$$


The last example is from renewal theory.

Example 4.3. (The renewal function for terminating renewal processes.) Let $T_{1}, T_{2}, \ldots$ be a sequence of nonnegative, independent, and identically distributed random variables with common distribution function $F$; we define the renewal counting process

$$
N(t)=\sum_{n=1}^{\infty} \mathbf{1}\left\{T_{1}+\cdots+T_{n} \leq t\right\}, \quad t \geq 0,
$$

where 1 is the indicator function. The function $M(t)=\mathrm{E} N(t)$ is called the renewal function. It gives the expected number of renewals in the interval $[0, t]$. To avoid trivialities we assume that the interrenewal distances $T_{1}, T_{2}, \ldots$ are not concentrated at zero, that is, $F(0)<1$. If $F(\infty)<1$ then the resulting renewal process is called terminating or transient. Then $M$ satisfies (see, e.g. Feller (1971)) the following defective renewal equation:

$$
M(t)=F(t)+\int_{0}^{t} M(t-x) \mathrm{d} F(x), \quad x \geq 0 .
$$

Clearly, (4.3) is of the form (1.1) with $\alpha=F(\infty)$ and $g(t)=F(t)$. Assume that $F \in \delta(\gamma)$, $\gamma \geq 0$. Then Theorem 3.2 yields

$$
M(\infty)-M(t) \sim \begin{cases}\frac{F(\infty)-F(t)}{(1-F(\infty))^{2}} & \text { if } \gamma=0, \\ \frac{F(\infty)-F(t)}{\left(1-m_{F}(\gamma)\right)^{2}} & \text { if } \gamma>0 .\end{cases}
$$

The result for the case $\gamma=0$ can be found in Teugels (1975, Theorem 4); here we have provided a different proof.

\section{Acknowledgements}

This research was supported by the National Natural Science Foundation of China (grant number 10471076), the Natural Science Foundation of Shandong (grant number Y2004A06), the Key Project of Chinese Ministry of Education (grant number 206091), and the Research Fund for the Doctoral Program of Higher Education (grant number 20050446001). The authors thank the Editor and an anonymous referee for helpful comments and suggestions on an earlier version of this paper.

\section{References}

ASMusSen, S. (1998). A probabilistic look at the Wiener-Hopf equation. SIAM Rev. 40, 189-201.

CAI, J. AND GARrido, J. (2002). Asymptotic forms and tails of convolutions of compound geometric distributions, with applications. In Recent Advances in Statistical Methods, ed. Y. P. Chaubed, Imperial College Press, London, pp. 114-131.

CAI, J. AND TANG, Q. (2004). On max-sum equivalence and convolution closure of heavy-tailed distributions and their applications. J. Appl. Prob. 41, 117-130.

Chover, J., Ney, P. And Wainger, S. (1973a). Degeneracy properties of subcritical branching processes. Ann. Prob. 1, 663-673.

Chover, J., Ney, P. And Wainger, S. (1973b). Functions of probability measures. J. Anal. Math. 26, $255-302$.

Cline, D. B. H. (1986). Convolution tails, product tails and domains of attraction. Prob. Theory Relat. Fields $\mathbf{7 2 ,}$ 529-557.

Cline, D. B. H. (1987). Convolutions of distributions with exponential and subexponential tails. J. Austral. Math. Soc. A 43, 347-365. 
Embrechts, P. AND Goldie, C. M. (1980). On closure and factorization properties of subexponential and related distributions. J. Austral. Math. Soc. A 29, 243-256.

Embrechts, P. And Goldie, C. M. (1982). On convolution tails. Stoch. Process. Appl. 13, 263-278.

Embrechts, P. And Veraverbeke, N. (1982). Estimates for the probability of ruin with special emphasis on the possibility of large claims. Insurance Math. Econom. 1, 55-72.

Embrechts, P., Goldie, C. M. and Veraverbeke, N. (1979). Subexponentiality and infinite divisibility. Z. Wahrscheinlichkeitsth. 49, 335-347.

Embrechts, P., KLÜPPelberg, C. And Mikosch, T. (1997). Modelling Extremal Events for Insurance and Finance. Springer, New York.

Feller, W. (1971). An Introduction to Probability Theory and Its Applications, Vol. 2, 2nd edn. John Wiley, New York.

Gerber, H. U. (1979). An Introduction to Mathematical Risk Theory. S. S. Huebner Foundation, University of Pennsylvania, Philadelphia.

Gerber, H. U. and Shiu, E. S. W. (1998). Pricing perpetual options for jump processes. N. Amer. Actuarial J. 2, $101-107$.

KLÜPPELBERG, C. (1988). Subexponential distributions and integrated tails. J. Appl. Prob. 25, 132-141.

Klüppelberg, C. (1989). Subexponential distributions and characterizations of related classes. Prob. Theory Relat. Fields 82, 259-269.

Rolski, T., Schmidli, H., Schmidt, V. And Teugels, J. (1999). Stochastic Processes for Insurance and Finance. John Wiley, New York.

Teugels, J. L. (1975). The class of subexponential distributions. Ann. Prob. 3, 1001-1011.

Willmot, G., CAi, J. And Lin, X. S. (2001). Lundberg inequalities for renewal equations. Adv. Appl. Prob. 33, 674-689. 